Brit. 7. Ophthal. (1974) 58, $75^{2}$

\title{
Influence of menstruation on corneal sensitivity
}

\author{
MICHEL MILLODOT AND ANNE LAMONT \\ Laboratory of Experimental Optometry, University of Montreal, Canada
}

Intraocular pressure has been shown by Dalton ( 1967$)$ to increase during the paramenstruum of women with closed-angle glaucoma. It is also known that there is a reduced corneal sensitivity with an increase in intraocular pressure as in glaucoma (Boberg-Ans, 1955). However, it is not known if corneal sensitivity of the healthy eye varies during the menstrual cycle and this study reports on the variations of the corneal touch threshold of young women during the menstrual cycle. We found a significant decrease in corneal sensitivity corresponding with the paramenstruum which was not found either in women taking oral contraceptives, or in men, who served as control groups.

\section{Material and methods}

The subjects were divided into three groups.

(I) Nine females, two of whom were tested daily and the remaining seven were asked to report 14 days and 24 hours before the onset of menstruation and again 14 days later.

(2) Five females taking oral contraceptives tested at the same 14-day intervals, except for one who was measured daily.

(3) A control group of eight males, one of whom reported daily and the others at 14-day intervals.

All subjects were free of ocular pathology and between 21 and 31 years of age, and all were tested on the right eye only.

The Cochet-Bonnet Aesthesiometer (Cochet and Bonnet, 1960), based on the instrument devised by Boberg-Ans (1955), was used to stimulate the cornea. Two models of the instrument, with nylon monofilaments of 0.12 and $0.08 \mathrm{~mm}$. diameter, were used to produce pressures of from I I to $200 \mathrm{mg}$./ $0.0113 \mathrm{~mm} .^{2}$ and from 2 to $90 \mathrm{mg} . / 0.005 \mathrm{~mm} .^{2}$ respectively. The aesthesiometer was mounted in a holder, allowing movement in $x, y$, and $z$ axes, so that reliability in stimulation of a corneal point, steady speed of application (Boberg-Ans, 1956), and a perpendicular corneal contact was achieved. A corneal point near the limbus in the 6 o'clock position was stimulated and the slightest bend of the nylon wire visible through a $\times 4.3$ magnifier was defined as corneal contact. All measurements were taken when the humidity was between 15 and 35 per cent. because the nylon monofilament is affected by humidity (Millodot and Larson, 1967). Subjects were tested at the same time of day to avoid confusion with the diurnal variation in corneal sensitivity (Millodot, 1972).

The touch threshold was determined subjectively (Millodot, 1973). The measurements began with stimulation of the cornea at the lowest pressure and continued in an ascending fashion. At each determined length of the nylon monofilament four to six contacts were made, with at least one blank to test subject reliability. The subject indicated when he felt the probe by pressing a bell. From these readings the touch threshold was defined as the length of the monofilament for which the subject responded for $5^{0}$ per cent. of the number of stimulations. This length was converted into pressure using the calibration curve of the instrument relating length and pressure previously established (Millodot, 1969). 


\section{Results}

EXPERIMENTAL GROUP

The measurements made on nine women in the middle of the menstrual cycle, 24 hours before the beginning of menstruation, and again 14 days later, are shown in Fig. I. Each data point represents the mean of the corneal touch threshold. The vertical lines show the standard deviations. The onset of menstruation did not always occur when expected and the hatched area in Fig. I indicates the range of the various onsets of menstruation for the group. The corneal touch thresholds increased markedly (i.e. lower sensitivity) during the premenstruum as compared to the values obtained during the intermenstruum. The mean corneal touch thresholds were $14.50,33^{\circ} 66$, and $14.94 \mathrm{mg} . / \mathrm{mm} .^{2}$ for the three occasions and the standard deviations were $7{ }^{\circ} 07,20^{\circ} 9$, and $8 \cdot 7 \mathrm{I}$ respectively. Hence the sensitivity decreased by 128 per cent. on the average during the premenstruum. This difference appeared in all subjects and the range of the decrease varied between 63 and 200 per cent. The difference between the mean of the first set of measurements and that of the premenstruum is significant $(t=3.73 ; \mathrm{P}<0 \cdot 005)$. The difference between the mean of the premenstruum and the third set of measurements is also significant $(t=4 \cdot 13 ; \mathrm{P}<0 \cdot 005)$.
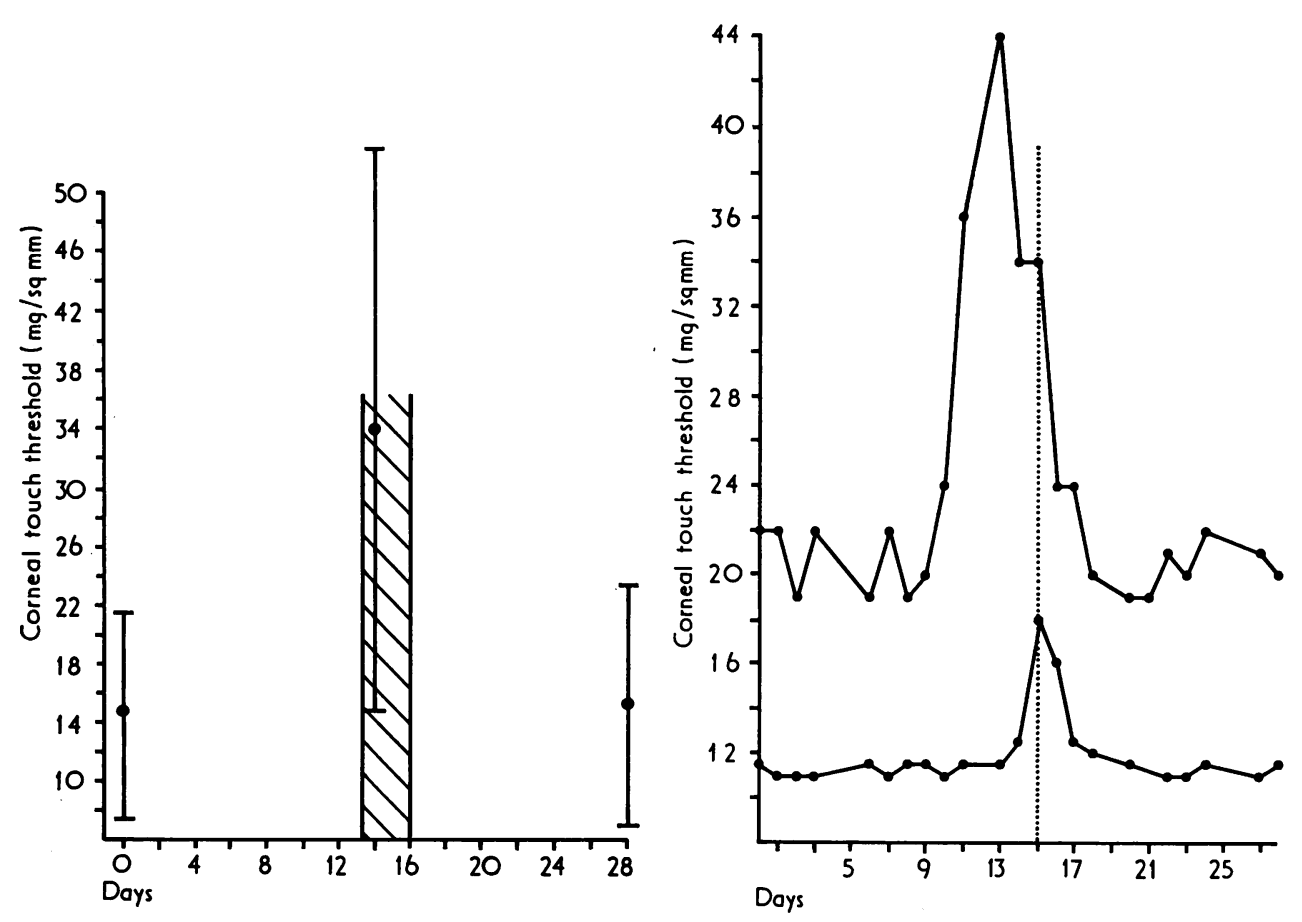

FIG. I Relationship between corneal touch threshold and number of days of menstrual cycle. Hatched area indicates range of onset of menstruation. Vertical lines represent standard deviations. Each data point is the mean of nine women

FIG. 2 Relationship between corneal touch threshold and number of days of menstrual cycle in two subjects. Dotted line represents onset of menstruation

Fig. 2 shows the readings obtained on two subjects throughout the cycle. The increase in threshold (or decrease in sensitivity) is obvious from these data; it lasts a few days around the onset of menstruation, indicated in Fig. 2 by the vertical line. 


\section{SEGOND EXPERIMENTAL GROUP}

The results for the five women taking oral contraceptives are given in Fig. 3. The lower line joins the mean data points obtained for this group on three occasions during the cycle. The hatched area represents the range of onset of menstruation. The mean value for each of the three data points is $10.9,10 \cdot 9$, and I I $\mathrm{mg} . / \mathrm{mm} .^{2}$, and the standard deviation is $\mathrm{r} .69$, $\mathrm{I} \cdot 2$, and $\mathrm{I} \cdot 68$ respectively. The upper line joins the readings obtained for one subject over a period of 28 days. The vertical dotted line marks the onset of menstruation for that subject. It is clear from these data that no discernible change in corneal touch threshold occurred during a menstrual cycle.
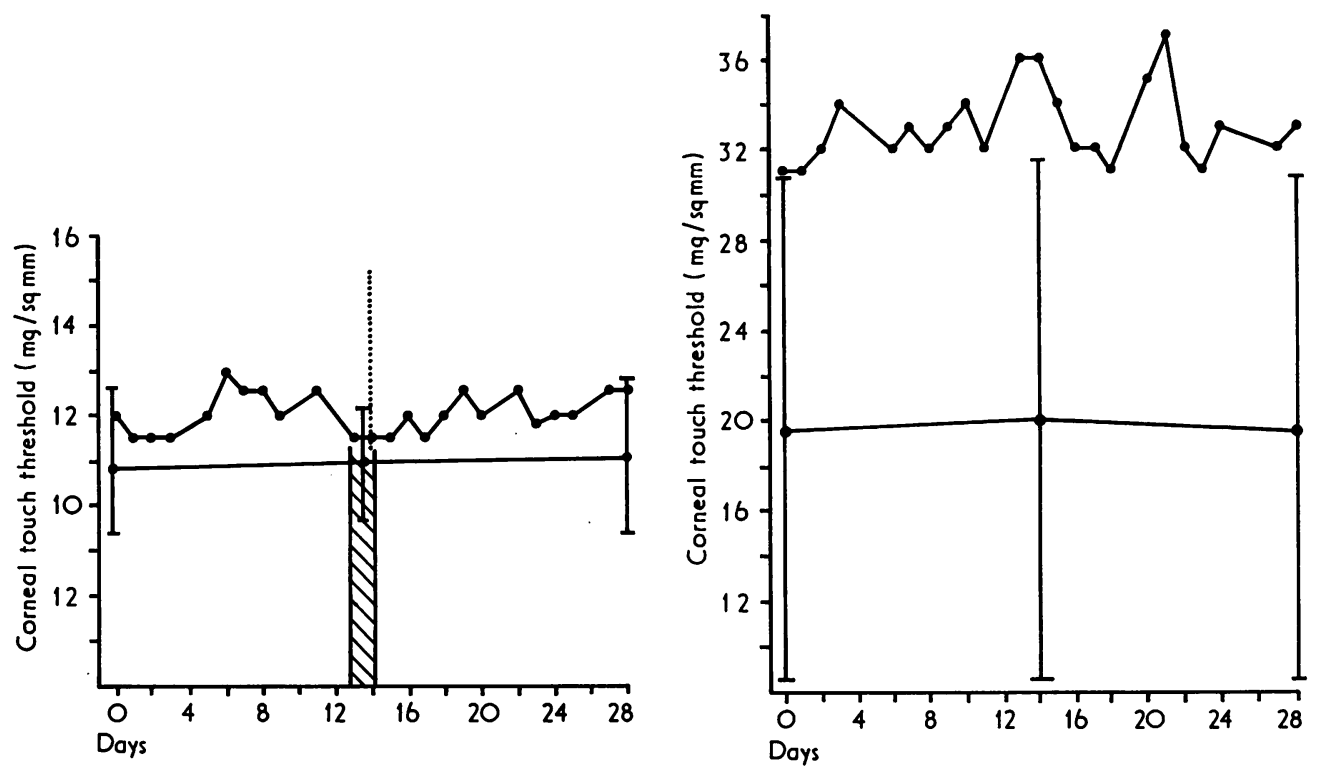

FIG. 3 Relationship between corneal touch threshold and number of days of menstrual cycle in women taking oral contraceptives. Lower line joins three data points which are the mean of five women. Hatched area indicates range of onset of menstruation. Vertical lines represent standard deviations. Upper curve joins data obtained on one subject throughout the menstrual cycle

FIG. 4 Relationship between corneal touch threshold in the course of 28 days. Lower line joins data points which are the mean of eight men. Vertical lines represent standard deviations. Upper curve ioins data obtained on one subject throughout the 28 days

\section{GONTROL GROUP}

The measurements for this group are shown in Fig. 4. The lower curve represents the mean data obtained on the eight men at three different times throughout a cycle of 28 days. The mean value for each of these three data points is $19.66,19.90$, and $19.50 \mathrm{mg} . / \mathrm{mm} .^{2}$ and the standard deviation is $I I \cdot 48, I I \cdot 75$, and $I I \cdot 05$ respectively. The upper curve shows the results obtained for one of these men throughout the cycle. It is clear that there is no systemic change in corneal touch threshold in the course of 28 days.

\section{Discussion}

This remarkable variation in corneal sensitivity during the menstrual cycle may be accounted for by the fact that after ovulation the body retains water; this retention is only 
relieved by the menstrual flow and this generalized oedema must also involve the cornea. That sensitivity is reduced during the premenstruum was also noted by Herren (1933), who recorded a diminution of tactile sensitivity of the finger, and this finding also could be attributed to the oedema present at that time. It is not known, however, whether the cornea becomes oedematous during the premenstruum. Bergin (1952) found a slightly larger dioptric power of the eye $(0.26 \mathrm{D})$ in six of seven subjects at or about the time of menstruation, and this most likely signifies an increase in corneal curvature due to oedema.

Dalton (1967) showed that the premenstruum was accompanied by a rise in intraocular pressure in glaucoma patients. A rise in intraocular pressure causes a reduction in corneal sensitivity (Boberg-Ans, 1955). Thus it is possible that both factors, water retention and an increase in intraocular pressure, may somehow contribute to this variation in corneal sensitivity. On the other hand the effect of contraceptive pills is to inhibit ovulation and to produce a more or less even physiological state throughout the cycle; when menstruation was not allowed no variation in corneal sensitivity was noted.

The results of the admittedly small group taking oral contraceptives show that these women are more sensitive than the other groups. This finding could be interpreted to mean that the eyes of women taking oral contraceptives are more sensitive and to this factor could be attributed the fact that some women find that their contact lenses become uncomfortable after they start taking the pill. Because of the small size of this group we have also measured the corneal sensitivity of five other women taking the pill. The mean corneal touch threshold for the total of ten subjects was found to be $14.15 \mathrm{mg} . / \mathrm{mm} .^{2}$ and the standard deviation 4.37. This mean value is slightly less than that obtained in the intermenstruum of the experimental group, but the difference is not statistically significant $(t=0.12 ; P>0.5)$. It is interesting to note that, of the women taking the pill, two were measured before taking the pill and their corneal sensitivity was the same. Obviously this problem must be further investigated.

The comparison between male and female corneal sensitivity becomes complex, as one must take into consideration the variation occurring throughout the female menstrual cycle. An average value for a group of females may not be a judicious means of evaluating female corneal sensitivity.

Not only is the probability of being involved in an accident greater during the premenstruum and menstruation phases (Dalton, I960), but the reduction in corneal sensitivity at that time makes women more vulnerable as any injury would be less noticeable. This information may be of value in contact lens practice, because adapting to lenses during this phase of the menstrual cycle may be most comfortable to the patient, but may be followed by hitherto incomprehensible disappointments after the sensitivity returns to its average level.

\section{Summary}

Corneal sensitivity was measured over a 28-day period in three groups of subjects: females, females taking contraceptive pills, and males. It was found that corneal sensitivity was reduced markedly during the premenstruum and about the time of menstruation as compared to the rest of the cycle. It remained about the same throughout the cycle in females taking contraceptive pills and in males. It is suggested that these results may be linked to the generalized oedema induced during the premenstruum, since no change in corneal sensitivity was recorded either in females taking oral contraceptives or in males in whom no such physiological variation is known to take place. 


\section{References}

Bergin, D. A. (I952) Amer. J. Optom., 29, I 29

BOBERG-ANS, J. (I955) Brit. F. Ophthal., 39, 705

(1956) Acta ophthal. (Kbh.), 34, I 49

Cochet, P., and Bonnet, R. (I96o) Clin. ophtal., 4, 3

DALTon, K. (I96o) Brit. med. 7., 2, I425

(1967) Brit. 7. Ophthal., 51, 692

herren, R. Y. (1933) 7 . exp. Psychol., I6, 324

MILloDot, M. (I969) Optician, I57, 267

(1972) Brit. F. Ophthal., 56, 844

(1973) Acta ophthal. (Kbh.), 51, 325

and LARson, w. ( 1967 ) Contact Lens (Lond.), r, 5 